\title{
An Optical Clock Based on a Single Trapped ${ }^{199} \mathrm{Hg}^{+}$lon
}

\author{
S. A. Diddams, ${ }^{1 *}$ Th. Udem, ${ }^{1} \dagger$ J. C. Bergquist, ${ }^{1}$ E. A. Curtis, ${ }^{1,2}$ \\ R. E. Drullinger, ${ }^{1}$ L. Hollberg, ${ }^{1}$ W. M. Itano, ${ }^{1}$ W. D. Lee, ${ }^{1}$ \\ C. W. Oates, ${ }^{1}$ K. R. Vogel, ${ }^{1}$ D. J. Wineland ${ }^{1}$
}

Microwave atomic clocks have been the de facto standards for precision time and frequency metrology over the past 50 years, finding widespread use in basic scientific studies, communications, and navigation. However, with its higher operating frequency, an atomic clock based on an optical transition can be much more stable. We demonstrate an all-optical atomic clock referenced to the 1.064-petahertz transition of a single trapped ${ }^{199} \mathrm{Hg}^{+}$ion. A clockwork based on a mode-locked femtosecond laser provides output pulses at a 1-gigahertz rate that are phase-coherently locked to the optical frequency. By comparison to a laser-cooled calcium optical standard, an upper limit for the fractional frequency instability of $7 \times 10^{-15}$ is measured in 1 second of averaging-a value substantially better than that of the world's best microwave atomic clocks.

Since the development of the first atomic clocks around 1950, it was recognized that the stability and accuracy of standards based on atomic transitions would benefit from choosing transition frequencies as high as possible. However, because it was not possible to count cycles of an oscillator at an arbitrarily high frequency in order to generate time, most time standards have been based on hyperfine transitions in atoms that occur at microwave frequencies where the period of one oscillation corresponds to roughly 0.1 to $1 \mathrm{~ns}$. In fact, since 1967 the internationally accepted definition of the second has been based on the $9,192,631,770-\mathrm{Hz}$ ground-state hyperfine transition in Cs, and Csbased clocks are currently the world's most accurate time standards with a fractional frequency uncertainty of about $1 \times 10^{-15}(1-4)$. Although the stability and accuracy of an optical clock based on a spectrally narrow atomic transition in the visible/ultraviolet (UV) region might be better, the frequency is about $10^{15} \mathrm{~Hz}$, where the period of one oscillation is on the order of $1 \mathrm{fs}$. Until recently, it was not practical to count such high frequencies without loss of cycles, but the introduction of mode-locked femtosecond lasers has now made it possible to conveniently and accurately divide optical frequencies to countable microwave or radio frequencies (5-10). The all-optical atomic clock reported here is based on a single, laser-cooled, trapped ${ }^{199} \mathrm{Hg}^{+}$ion and a femtosecond laser comb that provides the phase-coherent clock-

${ }^{1}$ Time and Frequency Division, National Institute of Standards and Technology, 325 Broadway, Boulder, CO 80305, USA. '2Department of Physics, University of Colorado, Boulder, CO 80309, USA.

*To whom correspondence should be addressed. Email: sdiddams@boulder.nist.gov

$\dagger$ Present address: Max-Planck-Institut für Quantenoptik, Hans-Kopfermann Strasse 1, 85748, Garching, Germany. work. Our optical clock demonstrates a fractional frequency instability of $\leq 7 \times 10^{-15}$ with $1 \mathrm{~s}$ of averaging - a value substantially better than that of the world's best microwave atomic standards - and promises accuracy that would be difficult to achieve with atomic clocks based on microwave transitions. This optical clock provides two distinct outputs: a comb of stable and accurate optical frequencies throughout the visible and near infrared, and a pulsed electronic signal at $1 \mathrm{GHz}$ that is phase-coherent with the optical frequency comb. The quantum-limited instability of the $\mathrm{Hg}^{+}$ion optical clock is expected to be around $1 \times 10^{-15} \tau^{-1 / 2}$, where $\tau$ is the averaging time measured in seconds, with an ultimate fractional frequency uncertainty approaching $10^{-18}(11)$.

Ultrastable and accurate optical metrology tools can be expected to provide an even finergrained view of the physical world, much as precision spectroscopy in the past 50 years has opened the door to an improved understanding of many fundamental aspects of atoms and molecules $(12,13)$. Of particular interest will be the continued application of optical frequency standards in spectroscopy and the improved determination of the fine structure constant $\alpha$ and the Rydberg constant $R_{\infty}(14)$. As measurement stability and accuracy improve, metrologists may find themselves in the unique position of being able to observe physical "constants" evolve in time (15). Indeed, laboratory tests on the possible divergence of clocks based on different atomic transitions already provide some of the most stringent constraints of the variation of $\alpha$. Other experiments of fundamental importance for which precision clocks/oscillators are of value include searches for variations in the isotropy of space, a preferred reference frame, and Lorentz and charge-parity-time (CPT) symmetry violation (16-20). From a technological standpoint, there is little dispute that stable and accurate microwave atomic clocks have greatly improved navigation and communications. It is likely that optical clocks of the future will have a similarly important impact.

Atomic clock basics. All clocks consist of two major components: some device that produces periodic events or "clock ticks," and some means for counting, accumulating, and displaying each tick. For example, the swing of a pendulum provides the periodic events that are counted, accumulated, and displayed by means of a set of gears driving a pair of clock hands. Similarly, in a quartz watch, the mechanical vibrations of a small quartz crystal are electronically detected, accumulated, and displayed to generate time. Atomic clocks add a third component: the resonance of a well-isolated atomic transition, which is used to control the oscillator frequency. If the frequency of the oscillator is made to match the transition frequency (i.e., the oscillator is locked to the atomic transition frequency) between two nondegenerate and unperturbed atomic states, then the time generated can have improved long-term stability and accuracy. For an atomic clock based on a microwave transition, high-speed electronics count and accumulate a defined number of cycles of the reference oscillator to mark a second of time. The basic concepts are the same for an atomic clock based on an optical transition at a much higher frequency. In this case the oscillator is a laser locked to an optical transition, but no electronic device exists that can count the very fast optical oscillations. For this purpose, a specialized frequency divider (commonly called a frequency chain) is required. Until very recently, optical-to-microwave frequency chains have been complicated, large-scale devices, requiring significant resources for operation (21). However, as described below, a femtosecond laser-based clockwork greatly simplifies this problem of directly counting the optical frequency.

All other factors being equal, a higher transition frequency can produce a more stable frequency standard. This is the principal advantage of an optical atomic clock over a microwave clock because the operating frequency is $\sim 100,000$ times higher, providing a finer division of time and thus potentially higher precision. This is seen in the Allan deviation $\sigma_{y}(\tau)$ which provides a convenient measure of the fractional frequency instability of a clock as a function of the averaging time $\tau$ (22). For an oscillator locked to an atomic transition of frequency $v_{0}$ and linewidth $\Delta v$,

$$
\sigma_{y}(\tau) \approx\left\langle\frac{\Delta v_{r m s}}{v_{0}}\right\rangle_{\tau} \approx \frac{\Delta v}{\pi v_{0}} \sqrt{\frac{T}{\tau N}}
$$

where $\Delta v_{\mathrm{rms}}$ is the measured frequency fluctuation, $N$ is the number of atoms, and $T$ is the cycle time (i.e., the time required to make a determination of the line center) with $\tau>T$. 


\section{RESEARCH ARTICLES}

This expression assumes that technical noise is reduced to a sufficiently small level such that the quantum-mechanical atomic projection noise is the dominant stability limit (23, $24)$. In this limit $\sigma_{y}(\tau)$ decreases as the square root of the averaging time for all clocks, so a 10 -fold decrease in the short-term instability leads to a 100-fold reduction in averaging time $\tau$ to reach a given stability and uncertainty. This point is particularly important if one ultimately hopes to reach a fractional frequency uncertainty of $10^{-18}$, which is the anticipated level for optical clocks. In this case, $\sigma_{y}(\tau) \leq 1 \times 10^{-15} \tau^{-1 / 2}$ is clearly desirable to avoid inordinately long averaging times.

Principles of the optical clock. Fundamental ideas and technical developments principally in three areas have brought us to the point where we are now able to demonstrate an optical frequency standard representative of clocks of the future: (i) the idea (25, 26) and demonstration of laser cooling of atoms $(27,28)$, (ii) the frequency stabilization of lasers (29-32), and (iii) the concept (33) and demonstration $(5-10)$ that femtosecond mode-locked lasers combined with nonlinear fibers can provide a simple, direct, and phasecoherent connection between radio frequencies and optical frequencies. Although most of these concepts have existed for some time, and preliminary demonstrations of optical clocks have even been made (34-36), only now have the techniques and tools advanced to the levels required for optical frequency standards to move beyond the benchmark results of the microwave standards. Trapped ions have been laser-cooled into the LambDicke regime to the zero-point energy limit of a trap potential (37), dramatically reducing the Doppler shifts and providing near-stationary, relatively unperturbed atomic references for extended observation times (11). Similarly, large numbers of neutral atoms that are suitable references for optical clocks are routinely laser-cooled and then trapped in magneto-optic traps (38-43). Stabilized lasers have now been demonstrated with linewidths less than $0.2 \mathrm{~Hz}(32,44)$ and with the center frequency reproducibly controlled for extended times at the levels of $|\Delta f / f|<10^{-15}$ (45). And finally, a practical method for measuring optical frequencies based on femtosecond laser technology has been developed (5-8) and its measurement uncertainty tested to a few parts in $10^{16}(9)$.

These advances are exploited in our optical clock, which consists of a stable continuous wave $(\mathrm{CW})$ laser oscillator that is frequency doubled and locked to a narrow UV transition of a single trapped and laser-cooled ${ }^{199} \mathrm{Hg}^{+}$ion. Thus stabilized, the frequency of the laser light is coherently divided down to lower frequencies by means of a femtosecond mode-locked laser that ultimately produces an electronic output at a frequency of $1 \mathrm{GHz}$. A simplified representation of the coherent relation between the optical frequency standard and the microwave pulses is shown in Fig. 1. The envelope of the pulse train is made synchronous with the optical phase of the CW laser with, in our case, $\sim 532,361$ optical cycles between pulses (46). The pulses thus provide "clock ticks" that are coher-

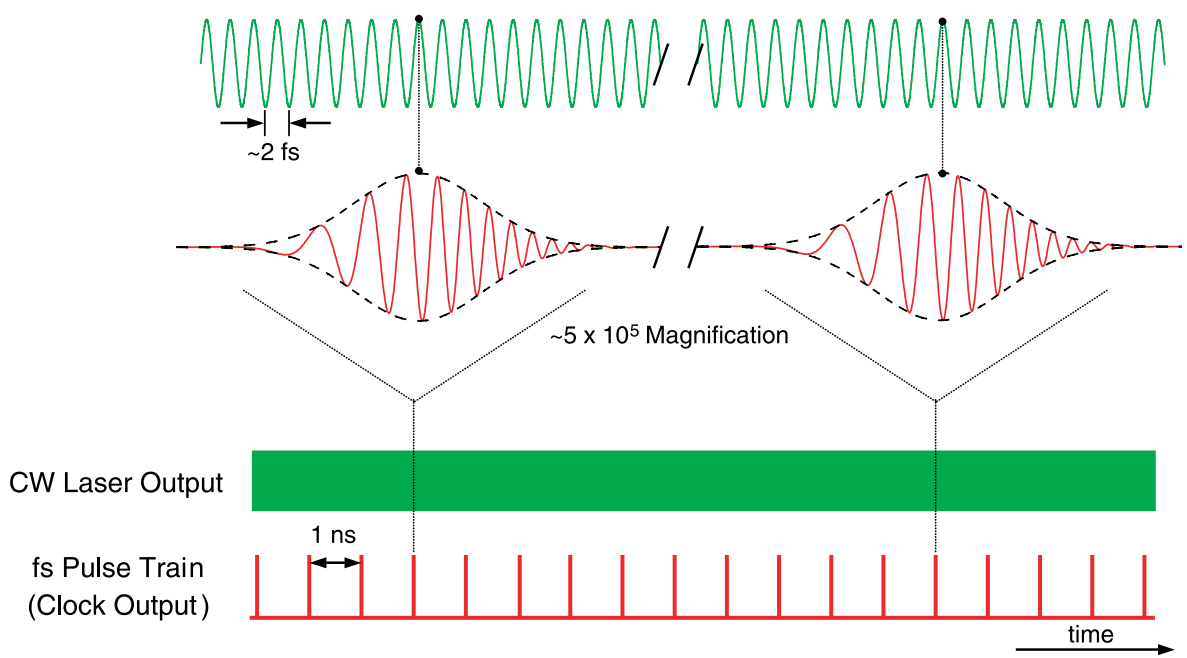

Fig. 1. Illustration of the time-domain relation between the atomically stabilized $532-\mathrm{THz} \mathrm{CW}$ laser field and the mode-locked femtosecond laser pulses. The two lines at the bottom show the near delta-function optical pulse train with a $f_{\mathrm{r}}=1-\mathrm{GHz}$ repetition rate and the $\mathrm{CW}$ laser output without the optical oscillation resolved. When the system is locked as an optically referenced femtosecond clockwork, the pulse repetition rate is phase-coherently related to the $532-\mathrm{THz} \mathrm{CW}$ field. This is indicated schematically in the upper portion of the figure where an expanded view of the lower two traces shows how the phase of the pulse envelope is related to the phase of the $\mathrm{CW}$ laser field. The net result is that the frequency of the pulse repetition rate is simply a rational fraction of the $\mathrm{Hg}^{+}$transition frequency.

ently connected to the $\mathrm{Hg}^{+}$transition.

The $\mathrm{Hg}^{+}$standard and optical clockwork. Figure 2 shows the optical clock in more detail, consisting of the optical frequency standard and the femtosecond laser-based optical clockwork. The ${ }^{199} \mathrm{Hg}^{+}$optical frequency standard has been described in detail elsewhere $(32,45)$, so we provide only the most relevant details. The ${ }^{2} \mathrm{~S}_{1 / 2}\left(\mathrm{~F}=0, \mathrm{M}_{\mathrm{F}}=\right.$ $0) \leftrightarrow{ }^{2} D_{5 / 2}\left(F=2, M_{F}=0\right)$ electric-quadrupole transition at $282 \mathrm{~nm}$ provides the reference for the optical frequency standard. The natural linewidth of the $\mathrm{S} \leftrightarrow \mathrm{D}$ resonance is about $2 \mathrm{~Hz}$ at $1.064 \mathrm{PHz}$, and recently a Fourier-transform-limited linewidth of only $6.7 \mathrm{~Hz}\left(Q=1.5 \times 10^{14}\right)$ has been observed (45). The "local oscillator" for the standard is the output of a well-stabilized 532-THz (563 $\mathrm{nm})$ dye laser $(32,44)$ that is frequency doubled and locked to the center of the 1.064$\mathrm{PHz}(282 \mathrm{~nm}) \mathrm{S} \leftrightarrow \mathrm{D}$ resonance. The shortterm ( 1 to $10 \mathrm{~s})$ fractional frequency instability of the probe laser is $\leq 5 \times 10^{-16}$. This short-term laser instability is low enough that information gathered from probing the ion transition can be integrated for about $10 \mathrm{~s}$ before it is used to steer the average laser frequency. The net result is a $\sigma_{y}(\tau) \leq 2 \times$ $10^{-15}$ for averaging times up to $\sim 30 \mathrm{~s}$, at which point $\sigma_{y}(\tau)$ begins to average down as $\tau^{-1 / 2}$.

The $\mathrm{Hg}^{+}$standard provides high accuracy and stability, but for distribution purposes and to realize a countable clock output, we must phase-coherently convert the optical signal to a lower frequency. The clockwork that divides the 1.064-PHz optical frequency to a countable microwave frequency $f_{\mathrm{r}}$ is based on a femtosecond laser and a novel microstructure optical fiber. The Ti:sapphire femtosecond ring laser emits a train of pulses (compressible to $\sim 25-\mathrm{fs}$ duration) at the nominal repetition rate of $f_{\mathrm{r}}=1 \mathrm{GHz}$ (47). The frequency-domain spectrum of the pulse train is a uniform comb of phase-coherent continuous waves separated by $f_{\mathrm{r}}$. The frequency of the $n$th mode of this comb is $f_{n}=$ $n f_{\mathrm{r}}+f_{\mathrm{o}}(48,49)$, where $f_{\mathrm{o}}$ is the frequency offset common to all modes that results from the difference between the group- and the phase-velocity inside the laser cavity. If the frequency comb of the laser covers an entire octave, then $f_{\text {o }}$ can be measured by frequency doubling an infrared mode $(n)$ and heterodyning it with an existing mode $(2 n)$ in the visible portion of the comb $(7,9)$. The heterodyne signal yields the frequency difference $2\left(n f_{\mathrm{r}}+f_{\mathrm{o}}\right)-\left(2 n f_{\mathrm{r}}+f_{\mathrm{o}}\right)=f_{\mathrm{o}}$. Only recently, with the arrival of microstructure silica fibers (also called photonic crystal fibers), has the required octave-spanning spectrum been attained with high repetition rate, low-power femtosecond lasers $(50,51)$. The unique dispersion properties of the microstructure fiber provide guidance in a single 


\section{RESEAR CHARTICLES}

spatial mode $(\sim 1.7-\mu \mathrm{m}$ diameter $)$ with zero group velocity dispersion near $800 \mathrm{~nm}(50)$. Because temporal spreading of the pulse is minimized, peak intensities in the range of hundreds of $\mathrm{GW} / \mathrm{cm}^{2}$ are maintained over a considerable propagation length, thus providing enhanced spectral broadening due to selfphase modulation. With $\sim 200 \mathrm{~mW}$ (average power) coupled into a $15-\mathrm{cm}$ piece of microstructure fiber, the total spectral width is broadened from $\sim 15$ to $\sim 300 \mathrm{THz}$ (spanning from $\sim 520 \mathrm{~nm}$ to $\sim 1170 \mathrm{~nm}$ ).

In addition to $f_{\mathrm{o}}$, a second heterodyne beat $f_{\mathrm{b}}$ is measured between an individual comb element $f_{m}=m f_{\mathrm{r}}+f_{\mathrm{o}}$ ( $m$ is an integer) and the 532-THz local oscillator of the $\mathrm{Hg}^{+}$standard. As shown in Fig. 2, two phase-locked loops (PLL) are used to control $f_{\mathrm{o}}$ and $f_{\mathrm{b}}$, thereby fixing the clock output $f_{\mathrm{r}}$. PLL-1 forces $f_{\mathrm{o}}=\beta f_{\mathrm{r}}$ by controlling the pump power of the femtosecond laser (9). Similarly, PLL-2 changes the cavity length of the femtosecond laser with a piezo-mounted mirror, such that $f_{\mathrm{b}}=\alpha f_{\mathrm{r}}$. The constants $\alpha$ and $\beta$ are integer ratios implemented with frequency synthesizers that use $f_{\mathrm{r}} / 100$ as a reference. In this manner, the frequencies of both PLLs are phase-coherently linked to $f_{\mathrm{r}}$ such that all oscillators used in the clock are referenced to the 532-THz laser oscillator itself. When $f_{\text {o }}$ and $f_{\mathrm{b}}$ are phase-locked, every element of the femtosecond comb, as well as their frequency separation $f_{\mathrm{r}}$, is phase-coherent with the laser locked to the $\mathrm{Hg}^{+}$standard (52). With no other frequency reference as an input, we realize all aspects of a high-accuracy, highstability optical atomic clock: a stable local oscillator (the laser) locked to a narrow atomic reference, and a pulsed microwave output that can be recorded with a counter.

High-stability output. With both PLLs closed, the $\sim 1-\mathrm{GHz}$ microwave output has the value of $f_{\mathrm{r}}=f_{\mathrm{Hg}} /(m \pm \alpha \pm \beta)$. If we choose the signs of beats $f_{\mathrm{o}}$ and $f_{\mathrm{r}}$ such that $\alpha=-\beta$, then $f_{\mathrm{r}}$ would be an exact subharmonic of $f_{\mathrm{Hg}}$. The stability of the 532-THz laser (given above) should be transferred to each element of the femtosecond comb, in addition to $f_{\mathrm{r}}$. We obtain $f_{\mathrm{r}}$ from the bandpassfiltered photocurrent generated with $\sim 5 \mathrm{~mW}$ of the broadened comb light incident on a p-i-n photodiode. We have measured the instability of $f_{\mathrm{r}}$ by subtracting it from the output of a synthesizer that is referenced to a hydrogen maser for which $\sigma_{v}(1 \mathrm{~s}) \approx 2.5 \times 10^{-13}$. The stability of this difference frequency is then analyzed with both a high-resolution counter and a dual-mixer time-measurement system (53). Both results are consistent with the resolutions of the respective measurements and the maser stability, demonstrating that the 1-s instability of $f_{\mathrm{r}}$ is at least as good as that of the hydrogen maser.

Before we can conclusively state that a microwave signal with stability matching that of the optical standard can be obtained from the optical clock, $f_{\mathrm{r}}$ needs to be compared to an oscillator with stability substantially better than that of the hydrogen maser. This could be either the microwave output of a second optical clock, or the high-stability output of a cryogenic microwave oscillator (54). Nonetheless, lacking these we can verify the exceptional stability of the comb in the optical sured stability with ac- domain and thereby infer the expected stability of $f_{\mathrm{r}}$. This is done by comparing one element of the optical comb to the Ca optical standard that operates at $456 \mathrm{THz}(657 \mathrm{~nm})$. For this measurement the femtosecond comb is phase-locked to the ${ }^{199} \mathrm{Hg}^{+}$standard as described above. For short averaging times, the femtosecond comb is effectively controlled by the stable Fabry-Perot cavity of the

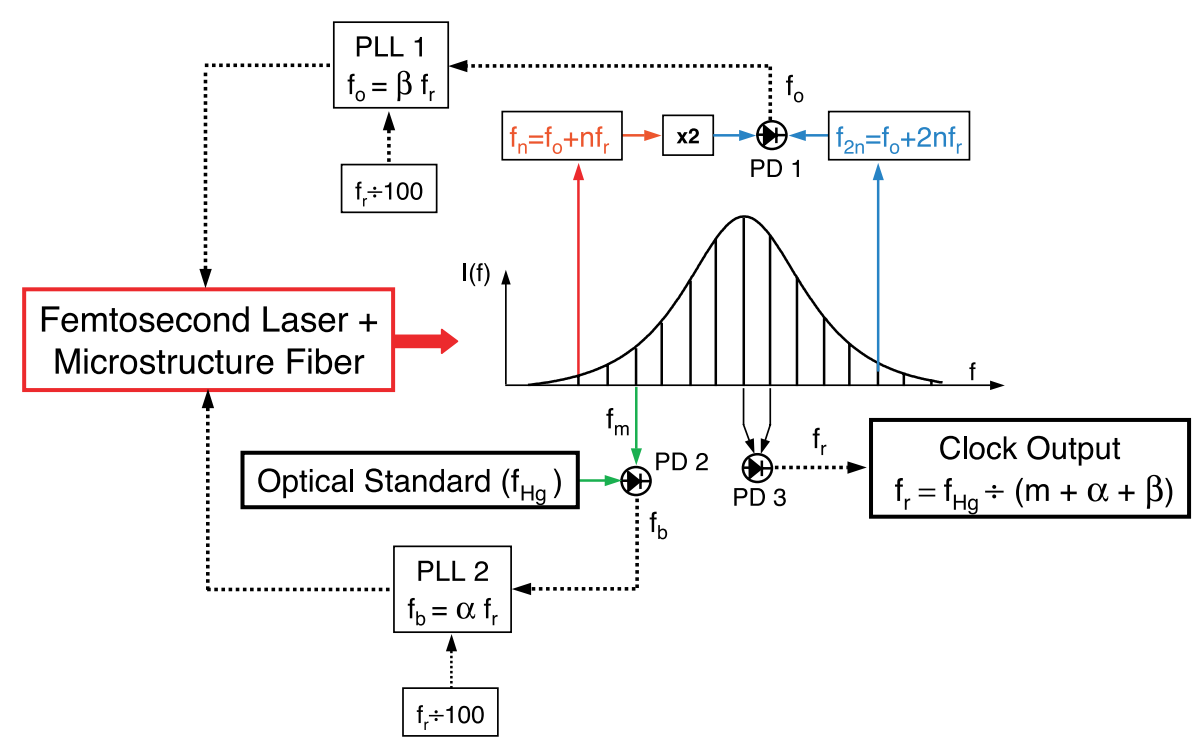

Fig. 2. Schematic of the self-referenced all-optical atomic clock. Solid lines represent optical beams, and dashed lines represent electrical paths. Photodiodes are designated by PD. The femtosecond laser, having repetition rate $f_{r}$ combined with the spectral broadening microstructure fiber produces an octave-spanning comb of frequencies in the visible/near infrared, represented by the array of vertical lines in the center of the figure. As shown above this comb, the low-frequency portion of the comb is frequency-doubled and heterodyned against the high-frequency portion in PD 1, yielding the offset frequency $f_{\mathrm{o}}$ that is common to all modes of the comb. Additionally, an individual element of the comb is heterodyned with the optical standard laser oscillator $\left(f_{\mathrm{Hg}}=532\right.$ $\mathrm{THz}$ ) that is locked to the clock transition frequency of a single ${ }^{199} \mathrm{Hg}^{+}$ion. When detected on PD 2 , this yields the beat frequency $f_{\mathrm{b}}$. Two phase-locked loops (PLL) control $f_{\mathrm{o}}$ and $f_{\mathrm{b}}$ with the result that the spacing $\left(f_{r}\right)$ of the frequency comb is phase-locked to the $\mathrm{Hg}^{+}$optical standard. Thus, $f_{r}$ is the countable microwave output of the clock, which is readily detected by illuminating PD 3 with the broadband spectrum from the frequency comb. See the text for further details.

Fig. 3. Measured stability of the heterodyne signal between one element of the femtosecond comb and the Ca optical standard at 456- THz (657 nm). The femtosecond comb is phase-locked to the $532-\mathrm{THz}$ laser oscillator. The black triangles are the stability data without cancellation of the additive fiber noise, which is represented by the dashed line. The red squares are the meative cancellation of the fiber noise and improved stability in the Ca standard. These results are about an order of magnitude better than the best stability reported with a Cs microwave standard, which is designated by the solid line (24). 
532-THz laser oscillator, and for $\tau \geq 30 \mathrm{~s}$ the control shifts to the ${ }^{199} \mathrm{Hg}^{+}$ion. We detect, filter, and count the heterodyne beat signal between a single element of the comb at 456 $\mathrm{THz}$ and a frequency-stabilized diode laser locked to the ${ }^{1} \mathrm{~S}_{0} \leftrightarrow{ }^{3} \mathrm{P}_{1}$ intercombination transition of a laser-cooled ensemble of $\mathrm{Ca}$ atoms $(43,55)$. The Bordé-Ramsey technique (56) is used for locking the diode laser to the 456- $\mathrm{THz}$ clock transition with resolutions ranging from 0.96 to $11.55 \mathrm{kHz}$, which are integer subharmonics of the recoil splitting. When the Ca standard is operated with 0.96$\mathrm{kHz}$ resolution, the Allan deviation of the heterodyne signal between the $\mathrm{Hg}^{+}$-stabilized comb and the $\mathrm{Ca}$ standard is shown as the triangles in Fig. 3. For $\tau<10 \mathrm{~s}$, the Allan deviation averages down roughly as $9 \times$ $10^{-15} \tau^{-1 / 2}$, which is consistent with the expected instability of the Ca standard in its present configuration. We also observe a monotonic degradation in the 1-s instability of the heterodyne beat frequency as the stability of the Ca standard is degraded by using lower resolution Ramsey fringes. However, for $\tau>10 \mathrm{~s}$, fluctuations introduced by the 180-m-long optical fiber that transmits the $532-\mathrm{THz}$ light to the femtosecond system begin to pose a limitation. We have measured the fiber-induced noise by double-passing the light through the optical fiber, and the average fractional frequency fluctuations are indicated by the dashed line in Fig. 3. Furthermore, for $\tau>30 \mathrm{~s}$, the instability of the $\mathrm{Hg}^{+}$ standard is anticipated to contribute to the measurement at approximately the same level as the $\mathrm{Ca}$ standard. Nonetheless, the measured stability improves with averaging to $\sim 1.5 \times 10^{-15}$ at $100 \mathrm{~s}$.

More recently, we have implemented active cancellation $(44,57)$ of this fiber noise and have further improved the signal-to-noise ratio in the Ca spectroscopy. Data taken under these conditions reveal a fractional frequency instability of $7 \times 10^{-15}$ at $\tau=1 \mathrm{~s}$. These results are plotted as the square data points in Fig. 3. In this case, we cannot place great significance in the stability for $\tau>1 \mathrm{~s}$ for two reasons. First, the Allan deviation for averaging times $\tau>1 \mathrm{~s}$ is calculated from the juxtaposition of 1-s averages. Such data analysis is known to result in biases for certain noise processes (58). Second, although not generally the case, for this specific data the 532-THz laser oscillator was not locked to the ${ }^{199} \mathrm{Hg}^{+}$ion, and therefore it was necessary to subtract out the smooth and predictable drift $(\sim 1 \mathrm{~Hz} / \mathrm{s})$ of the Fabry-Perot cavity to which this laser is stabilized. However, neither of these affect the measured 1-s Allan deviation, which provides an upper limit for the short-term (1-s) instability of $7 \times 10^{-15}$ for the optical comb. Again, this 1-s instability is consistent with that of the $\mathrm{Ca}$ standard in its present configuration. Similar stability in the $\sim 1$-GHz clock output remains to be verified.

\section{RESEARCH ARTICLES}

Conclusion. We have constructed an optical clock based on the 1.064-PHz $(282 \mathrm{~nm})$ electric-quadrupole transition in a lasercooled, single ${ }^{199} \mathrm{Hg}^{+}$ion. The optical frequency is phase-coherently divided down to provide a coherent microwave output through the use of a mode-locked femtosecond laser and a microstructured optical fiber. The short-term (1-s) instability of the optical output of the clock is measured against an independent optical standard to be $\leq 7 \times 10^{-15}$. This optically referenced femtosecond comb provides a countable output at $1 \mathrm{GHz}$, which should ultimately be usable as a higher accuracy reference for time scales, synthesis of frequencies from the radio frequency to the UV, comparison to other atomic standards, and tests of fundamental properties of nature.

\section{References and Notes}

1. P. Laurent et al., in Laser Spectroscopy XIV, R. Blatt, J. Eschner, D. Leibfried, F. Schmidt-Kaler, Eds. (World Scientific, Singapore, 1999), pp. 41-50.

2. S. R. Jefferts, D. M. Meekhof, J. H. Shirley, M. Stepanovic, T. E. Parker, in Proceedings of the 2000 IEEE International Frequency Control Symposium, Kansas City, 7 to 9 June 2000 (IEEE, Piscataway, NJ, 2000), pp. 714-717.

3. S. Weyers, R. Schröder, A. Bauch, in Proceedings of the 15th European Frequency and Time Forum, Neuchâtel, Switzerland, 6 to 8 March 2001 (Fondation Suisse pour la Recherche en Microtechnique, Neuchâtel, Switzerland, in press).

4. S. Weyers, U. Hübner, R. Schröder, Chr. Tamm, A. Bauch, Metrologia 38, 4 (2001).

5. Th. Udem, J. Reichert, R. Holzwarth, T. W. Hänsch, Phys. Rev. Lett. 82, 3568 (1999).

6. J. Reichert et al., Phys. Rev. Lett. 84, 3232 (2000).

7. D. J. Jones et al., Science 288, 635 (2000).

8. S. A. Diddams et al., Phys. Rev. Lett. 84, 5102 (2000)

9. R. Holzwarth et al., Phys. Rev. Lett. 85, 2264 (2000)

10. J. Stenger et al., Phys. Rev. A 63, 021802R (2001).

11. A. A. Madej, J. E. Bernard, in Frequency Measurement and Control, A. N. Luiten, Ed. (Springer-Verlag, Berlin, 2001), pp. 153-194.

12. See, for example, J. L. Hall, Colloques Internationaux du C.N.R.S. No. 217-Méthodes de Spectroscopie Sans Largeur Doppler de Niveaux Excités de Systèmes Moléculaires (CNRS, Paris, 1974), pp. 105-125.

13. T. W. Hänsch, A. L. Schawlow, G. W. Series, Sci. Am 240, 72 (March 1979).

14. See, for example, the contributions of $F$. Biraben et al. (pp. 17-41) and Th. Udem et al. (pp. 125-144), in The Hydrogen Atom: Precision Physics of Simple Atomic Systems, S. V. Karshenboim, F. S. Pavone, F. Bassani, M. Inguscio, T. W. Hänsch, Eds. (Springer-Verlag, Berlin, 2001).

15. S. V. Karshenboim, Can. J. Phys. 78, 639 (2000).

16. A. Brillet, J. L. Hall, Phys. Rev. Lett. 42, 549 (1979).

17. M. P. Haugen, C. M. Will, Phys. Today 40, 69 (1987).

18. D. Hils, J. L. Hall, Phys. Rev. Lett. 64, 1697 (1990).

19. V. A. Kostelecky, Ed. CPT and Lorentz Symmetry (World Scientific, Singapore, 1999).

20. D. Bear, R. E. Stoner, R. L. Walsworth, V. A. Kostelecky, C. D. Lane, Phys. Rev. Lett. 85, 5038 (2000).

21. See, for example, C. O. Weiss, G. Kramer, B. Lipphardt, $\mathrm{H}$. Schnatz, in Frequency Measurement and Control, A. N. Luiten, Ed. (Springer-Verlag, Berlin, 2001), pp. 215-247.

22. J. A. Barnes et al., IEEE Trans. Instrum. Meas. 20, 204 (1971).

23. W. M. Itano et al., Phys. Rev. A 47, 3554 (1993).

24. G. Santarelli et al., Phys. Rev. Lett. 82, 4619 (1999).

25. T. W. Hänsch, A. L. Schawlow, Opt. Commun. 13, 68 (1975).

26. D. J. Wineland, H. G. Dehmelt, Bull. Am. Phys. Soc. 20, 637 (1975).

27. D. J. Wineland, W. M. Itano, Phys. Today 40, 34 (1987).
28. C. Cohen-Tannoudji, W. D. Phillips, Phys. Today 43, 33 (1990).

29. J. L. Hall, Science 202, 147 (1978).

30. Ch. Salomon, D. Hils, J. L Hall, J. Opt. Soc. Am. B, 8, 1576 (1988).

31. S. N. Bagaev, Yu. D. Kolomnikov, V. N. Lisitsyn, V. P. Chebotayev, IEEE J. Quant. Electr. 4, 868 (1968).

32. B. C. Young, F. C. Cruz, W. M. Itano, J. C. Bergquist, Phys. Rev. Lett. 82, 3799 (1999).

33. J. N. Eckstein, A. I. Ferguson, T. W. Hänsch, Phys. Rev. Lett. 40, 847 (1978).

34. V. P. Chebotayev et al., Appl. Phys. B 29, 63 (1982).

35. C. O. Weiss, G. Kramer, B. Lipphardt, E. Garcia, IEEE J. Quant. Electr. 24, 1970 (1988).

36. J. Ye, J. L. Hall, S. A. Diddams, Opt. Lett. 25, 1675 (2000).

37. F. Diedrich, J. C. Bergquist, W. M. Itano, D. J. Wineland, Phys. Rev. Lett. 62, 403 (1989).

38. T. Kurosu, F. Shimizu, Jpn. J. Appl. Phys. 29, L2127 (1990).

39. Th. Kisters, K. Zeiske, F. Riehle, J. Helmcke, Appl. Phys. B 59, 89 (1994).

40. F. Ruschewitz et al., Phys. Rev. Lett. 80, 3173 (1998).

41. H. Katori, T. Ido, Y. Isoya, M. Kuwata-Gonokami, Phys. Rev. Lett. 82, 1116 (1999)

42. K. R. Vogel, T. P. Dinneen, A. Gallagher, J. L. Hall, in Proceedings of the Joint Meeting of the European Frequency and Time Forum and the IEEE International Frequency Control Symposium, Besançon, France, 13 to 16 April 1999 (IEEE, Piscataway, NJ, 1999), Pp. 692-695.

43. C. W. Oates, F. Bondu, R. W. Fox, L. Hollberg, J. Phys. D 7, 449 (1999)

44. B. C. Young et al., in Laser Spectroscopy XIV, R. Blatt, J. Eschner, D. Leibfried, F. Schmidt-Kaler, Eds. (World Scientific, Singapore, 1999), pp. 61-70.

45. R. Rafac, et al., Phys. Rev. Lett. 85, 2462 (2000).

46. The coherent relation between the pulse envelope and the $532-\mathrm{THz}$ CW laser oscillator is established by first phase-locking the rate $f_{\mathrm{o}}$ at which the pulse carrier advances with respect to the envelope from pulse to pulse. For illustrative purposes in Fig. 1, we have chosen this rate to be $f_{f}=f_{\mathrm{r}} / 10$, such that every 10th pulse is identical. The pulse envelope is then made phase coherent with the CW laser oscillator by detecting and controlling the heterodyne beat between the CW laser and the carrier field of the pulsed laser. The frequency domain description is given in the text.

47. A. Bartels, T. Dekorsy, H. Kurz, Opt. Lett. 24, 996 (1999).

48. A. I. Ferguson, J. N. Eckstein, T. W. Hänsch, Appl. Phys. 18, 257 (1979).

49. J. Reichert, R. Holzwarth, Th. Udem, T. W. Hänsch, Opt. Commun. 172, 59 (1999).

50. J. K. Ranka, R. S. Windeler, A. J. Stentz, Opt. Lett. 25, 25 (2000)

51. W. J. Wadsworth et al., Electron. Lett. 36, 53 (2000).

52. We count the phase-locked beats $f_{\text {ond }}$ an with a highresolution counter and verify that they fluctuate about their respective phase-locked values by less than 100 $\mathrm{mHz}$ in $1 \mathrm{~s}$. This implies that the stability of the $532-\mathrm{THz}$ laser oscillator is transferred to the femtosecond comb with a relative uncertainty $<2 \times 10^{-16}$ in $1 \mathrm{~s}$.

53. S. Stein et al., in Proceedings of the 1982 IEEE International Frequency Control Symposium, Philadelphia, PA, 2 to 4 June 1982 (IEEE, Piscataway, NJ, 1982), pp. 24-30.

54. S. Chang, A. G. Mann, A. N. Luiten, Electron. Lett. 36, 480 (2000).

55. C. W. Oates, E. A. Curtis, L. Hollberg, Opt. Lett. 25 1603 (2000).

56. C. J. Bordé, Phys. Lett. A 140, 10 (1989).

57. L.-S. Ma, P. Jungner, J. Ye, J. L. Hall, Opt. Lett. 19, 1777 (1994).

58. P. Lesage, IEEE Trans. Instrum. Meas. 32, 204 (1983).

59. We are grateful to A. Bartels (GigaOptics $\mathrm{GmbH}$ ) for assistance with the femtosecond laser. We are also indebted to R. Windeler (Lucent Technologies) for providing the microstructure optical fiber. We acknowledge many illuminating discussions with J. Hall, S. Cundiff, J. Ye, S. Karshenboim, and F. Walls. Contribution of the National Institute of Standards and Technology-not subject to U.S. copyright.

29 March 2001; accepted 11 June 2001 Published online 12 July 2001;

10.1126/science.1061171

Include this information when citing this paper. 